\title{
Weekend effect: a great problem with a potential solution
}

\author{
Ana Abella ${ }^{1}$, Beatriz Lobo-Valbuena ${ }^{{ }^{*}}$ (D), Marcela Hómez ${ }^{1}$ and Federico Gordo ${ }^{1,2}$ \\ See related research by Zajic et al. https://ccforum.biomedcentral.com/articles/10.1186/s13054-017-1812-0
}

We have read with great interest the article by Zajic et al. [1] showing that patients admitted to the intensive care unit (ICU) on weekends had a higher mortality rate.

Recently, in a study of by group we found similar results; factors independently associated with hospital mortality were the Simplified Acute Physiology Score (SAPS) 3 (odds ratio (OR) 1.10; 95\% confidence interval (CI) 1.08-1.12) and belonging to the "offhours" group (weekend and holidays; OR 2.00; 95\% CI 1.20-3.33) [2].

We firmly believe in the priority of early detection of patients at risk of poor outcome, not only of the so-called "time-dependent" pathologies such as sepsis, stroke or ischemic heart disease, where early intervention can improve prognosis and reduce onset complications, but also including all potential ICU patients. For this reason, we have implemented since 2008 the "ICU without walls" protocol, which allows early detection of high-risk patients using a computer data alert system based on laboratory data [3].

With the assumption that worse prognosis may be related to the late detection of these patients, the "ICU without walls" protocol was developed also during weekends. Through a multivariate analysis, SAPS3 (OR 1.08, 95\% CI 1.06-1.11) and being part of the intervention group (OR 0.33, 95\% CI 0.12-0.89) were independently associated with mortality [4].

We would also like to emphasize that in the publication by Zajic and co-authors [1], patients admitted after scheduled surgery presented a lower ICU death risk (hazard ratio 0.43; 95\% CI 0.39-0.46). Not withdrawing scheduled surgery patients or not differentiating them from unplanned/urgent admissions could imply a selection bias, despite a baseline

\footnotetext{
* Correspondence: b.lobo.valbuena@gmail.com

${ }^{1}$ Intensive Care Unit, Hospital Universitario del Henares, Avenida María de

Curie s/n. 28822, Coslada, Madrid, Spain

Full list of author information is available at the end of the article
}

mortality risk adjustment using the SAPS3. Moreover, the difference in reasons for ICU admission between weekdays and weekends is noteworthy, as Zajic et al. discuss.

Finally, we would like to point out that the early detection of patients at risk of organ failure is a task that must be adapted to the circumstances of each center. A multidisciplinary team coordinated by the intensivist, with the help of electronic and organizational resources, may help to resolve this issue. Regarding this topic, Durie et al. [5] demonstrate good clinical results after applying a multidisciplinary approach, named "Code-ICU", dedicated to properly identifying candidates for ICU admission, displaying a reduction in ICU length of stay and hospital length of stay.

\section{Abbreviations \\ ICU: Intensive care unit; OR: Odds ratio; SAPS: Simplified Acute Physiology Score}

\section{Acknowledgements}

Not applicable.

\section{Funding}

No funding was required.

\section{Availability of data and materials \\ Yes}

\section{Authors' contributions}

$A B, B L V, M H$, and FD helped on manuscript preparation, and attest to the integrity of the data reported on the manuscript. All authors read and approved the final manuscript.

\section{Ethics approval and consent to participate Not applicable.}

\section{Consent for publication \\ Not applicable.}

\section{Competing interests}

The authors declare that they have no competing interests. 


\section{Publisher's Note}

Springer Nature remains neutral with regard to jurisdictional claims in published maps and institutional affiliations.

\section{Author details}

Intensive Care Unit, Hospital Universitario del Henares, Avenida María de Curie s/n. 28822, Coslada, Madrid, Spain. ²Universidad Francisco de Vitoria, Facultad Ciencias de la Salud, Edificio E, Ctra. M-515 Pozuelo-Majadahonda, Madrid, Spain.

Received: 4 October 2017 Accepted: 19 October 2017

Published online: 17 November 2017

\section{References}

1. Zajic P, Bauer P, Rhodes A, Moreno R, Fellinger T, Metnitz B, et al. Weekends affect mortality risk and chance of discharge in critically ill patients: a retrospective study in the Austrian registry for intensive care. Crit Care. 2017;21(1):223

2. Abella A, Hermosa C, Enciso V, Torrejon I, Molina R, Diaz M, et al. Effect of the timing of admission upon patient prognosis in the intensive care unit: on-hours versus off-hours. Med Intensiva. 2016;40(1):26-32.

3. Abella Alvarez A, Torrejon Perez I, Enciso Calderon V, Hermosa Gelbard C, Sicilia Urban JJ, Ruiz Grinspan M, et al. ICU without walls project. Effect of the early detection of patients at risk. Med Intensiva. 2013;37(1):12-8.

4. Abella A, Enciso V, Torrejon I, Hermosa C, Mozo T, Molina R, et al. Effect upon mortality of the extension to holidays and weekends of the "ICU without walls" project. A before-after study. Med Intensiva. 2016;40(5):273-9.

5. Durie ML, Darvall JN, Hadley DA, Tacey MA. A "Code ICU" expedited review of critically ill patients is associated with reduced emergency department length of stay and duration of mechanical ventilation. J Crit Care. 2017:42:123-8. 